\title{
Notes on the clinical practice of informational medicine
}

\begin{abstract}
This work will bring together information gleaned from the experience of the author in a clinical setting and the formal knowledge of Homeopathy, Traditional Chinese Medicine (TCM), the use of the cold laser, Electro-Acupuncture according to Dr. Voll (EAV) and Astrology. The information presented will be along the lines of a formal patient intake, to a discussion of signs \& symptoms and then to treatment modalities.
\end{abstract}

Keywords: astrology, chinese, medicine, homeopathy, laser, eav, patient, intake, environment, clinic, dowsing, body, field, harmonics, water, food, therapy, symptoms, meridians, treatment, anchoring, disease
Volume I Issue 5 - 2015

\author{
Edward F Block \\ Block Institute for Astrobiological Studies, USA
}

Correspondence: Edward F Block, Block Institute for Astrobiological Studies, 375 Dula Springs Road,Weaverville, North Carolina, USA, Email efblockiv@yahoo.com

Received: June 09, 2015 | Published: September 10,2015
Abbreviations: HBF, human body field; IM, informational medicine

\section{Clinical setting}

The concept of providing a neutral space for the practice of healing modes is very important. Careful planning on the part of the clinician will need to be done along several lines of investigation. The difficulty of finding a commercial space in which to practice your profession is daunting in itself. However, you will need to be very aware of the geomagnetic/Birkland current factors in the local setting (see Environmental Influences below). Perhaps you may learn to dowse and scope out the proposed clinical space yourself. If you feel uncomfortable in the proposed space, do not practice there.

It is suggested that you use only battery powered equipment in the immediate clinical setting. It is especially required to eliminate any electrical motors from the clinical setting such as fans. It would also be a very good idea to keep the treatment table at least 6 feet away from any electrical power outlet. ${ }^{1}$ Use your dowsing skills to find the location in the room that you are going to treat your patients where the Birkland current 3D grid is most neutral. Wooden floors are better than concrete. Try to get a room that is over an earthen space with a wooden floor, perhaps a building intended to originally be a home.

You the clinician will be in the space of the patient and your $\mathrm{HBF}^{2}{ }^{2}$ will interact with that of the patient and vice versa. Thus you will need to learn how to maintain your centered state of beingness. Washing your hands in running water aids in keeping adverse energy/ fields from accumulating in your aura. This is because clean water has a resonance at the Birkland harmonic of $4.55 \mathrm{HZ}$. Showers are better than baths since the negative energies go down the drain instead of remaining in the tub with you. It helps to take a minute between patients to deep breath, ground yourself and zone out to monitor your aura (HBF)

\section{Patient intake}

This aspect of patient care is perhaps the most important part of the whole entire process. Without a through understanding of the parameters involved in the patient related to their disease, nothing but in a general sense that you do for them will bring their HBF into alignment and effect a "cure". Please remember that you need to be familiar with their astrological natal chart, their lifestyle, their nutritional status, their environmental conditions and their interpersonal interactions. Thus, the question and answer portion of your interaction with the patient is critical. A good intake discussion should take about 2 hours. You will also need to enlighten them as to the nature of your treatment schedule and their need for strict adherence to your treatment protocol. Many people will not be willing to give up their lifestyle and are doomed thereby to failure in achieving any long lasting beneficial results.

\section{Astrology}

The very first thing that the author asks for is the date, place and time (hopefully within 4 minutes of birth but any information is helpful in getting the relative position of the Moon at the time of birth) of birth as that means that the most accurate astrologial Natal chart may be calculated. Please remember that Astrology is a representation of your genetic complement in physical form, emotional make up and mental propensities. You will need to shop around until you find the software program that suits your needs. The free online offerings are not detailed enough unless you have a great deal of astrological experience under your belt. I am not going to go into detail here but will give only the basics that are needed to be look at with care. Also, please remember that Astrology is reprentational and not actually causal. It is the inherited gene sets that are causal. ${ }^{3}$

The position and placement in the chart of the Moon is critical as the Moon is the generalized representation of your entire physical body. The relationship of the Moon placement with the other aspects of the Natal chart will give to you the general strengths and weaknesses of the physical body.

The Ascendant is the manner in which you project yourself into your environment. The Descendant is the energy that you cultivate in order to project yourself. Thus you need to pay attention to the relationship of the Descendant with the other aspects of the Natal chart as this will guide you in understanding the role of unconscious elements in the psyche that are at work.

The positions of Venus and Mars in the chart relates to the manner in which you receive physical energy from others and how you project your physical energies towards others. This dual relationship is what people call "vibes" and denotes how the patient will react to being 
close to another person over a long period of time. The people or person that you spend a great deal of time with has a definite effect upon the flow of energies within a person's aura (HBF). ${ }^{4}$

The planet Jupiter is the major governor of the emotions. The planet Mercury is the major governor of mentation. The planet Saturn is the governor of overall learning, growth and development. The planets Neptune, Uranus and the planetoid Pluto generally appear to fine tune any aspects of the other planets and their chart positions. A Natal chart will give to you confirmation of the information that the patient should give to you as you query their normal body functions. The Natal chart will also give to you clues as to what questions you should ask in order to cue the patient for their responses.

\section{Healthy (normal) physical body nuances}

The intake form upon which you record the answers that your patient gives to you concerning the status of their normal bodily functions may be found online. However, you will need to modify the sheet(s) of the form that suits your needs to reflect the manner in which you practice your clinical skills, i.e. you will need to tweak the form.

If you get a detailed description of the family history of disease patterns, this will give to you a general overview of the genetic predispositions that the patient carries. The family health history and individual astrological Natal chart analysis will give you a comprehensive background from which to develop and fine tune the treatment protocol for your respective patient, regardless of their present condition.

Obviously, the normal nuances of their body are required so as to discover the organ systems and channels that are involved in the HBF aberration (s) according to the manifest signs and symptoms. Some of your form modifications will be that of adding mental and emotional aspects to detail. This is where your knowledge of Homeopathy will come into play. It is definitely true that mental/emotional disturbances always proceed those of physical aberrations.

\section{Nutritional status and food therapy}

The diet of your patient needs to be detailed and may involve giving your patient forms to take home so as to record the time, types, combinations and amount of the foods consumed. This will assist your determination of any Food Therapy that you might prescribe for your patient. Again, it will also help if you have them record the times of consumption. This will fit into the TCM 24hour clock of Organ/Qi cycle and determine the times for your patient to consume the foods that you have prescribed according to the channel(s) affected. The herbal elements of the pharmacopeia always have a channel as point of entry into the energetic system of the HBF and thus dovetail with the 24 hour Organ/Qi clock system.

This is also where you will be prescribing herbal formulas for the immediate elimination of symptoms. However, herbal formulas may be prescribed in order to assist the body in dealing with toxins and waste products. The liver is the organ that metabolizes all of the substances absorbed by the gastro-intestinal tract and builds all of the non-cellular components of the blood..$^{5}$ As the liver is in the lower Jiao, dampness in the lower Jiao is particularly stressful. Herbal formulations tailored to the signs \& symptoms of the lower Jiao go a long way in assisting the liver in eliminating the causal factors of Dampness. This is also where the effects of the eating habits of your patients come to the fore. This means that you will need to become a skilled nutritionist as well. Also, even in ancient times, the more sophisticated and educated Chinese preferred to use the herbal pharmacopeia than the more crude acupuncture and bloodletting for a healing modality. ${ }^{6}$

\section{Lifestyle}

Many people have food addictions and will be reluctant to give up the foods that have contributed or might contribute to their predicament. Thus, you will need to be able to coach them through their withdrawal and rehabilitation if they truly want to kick their habit(s) and prevent their conditions from becoming worse. They really will not become well if they do not change their lifestyle choices.

Also, your patient may have addictions to alcohol, nicotine, caffeine, prescribed medications and illegal substances. In reference to the last 2, your patient may be in denial or simply not tell the truth concerning their addiction. The patient may not come square with you until they have engendered a feeling of trust with you.

Home circumstances, work situations, school issues and a mydiad of other environmental factors will need to be detailed. All will play a role in the manner in which the patient copes with their lives and the types of stress encountered daily.

You might want to introduce your patient to the various means of stress reduction such as meditation, taking a walk, cat napping and listening to relaxing music. Get your patient creatively involved with their own rehabilitation. They will need to take ownership of their life circumstances before they can begin to make needed changes. You must ask the patient about their sleeping arrangements and how their sleep progresses through the night. This is critical in cases of patients having been diagnosed with cancer. It will be necessary to dowse the sleeping area and re-arrange the bed in the bedroom to reflect the nature of the 3D Birkland current matrix. In TCM, the use of feng shui is used to assist in this task.

\section{Environmental influences affecting the patient}

This is very important and most practitioners do not have the experience to ask the correct questions of their patients concerning the parameters involved. ${ }^{8}$ Inevitably, it is the phenomena of geopathic stressors that needs to be discovered and neutralized. Electrical motors are extremely harmful due to the adverse electromagnetic factors that are produced by them and sprayed into the local environment. In hospitals, it was empirically determined that all beds needed to be at least 6 feet apart so that patient illness did not adversely affect another patient. This is due to the influence of the state of the HBF of one person on that of another person.

As you may grasp, there are 3 major contributors to the environmental setting:

a. geomagnetic parameters of the local earthen crust and the Birkland currents that determine the generalized background

b. The electrical mains in situ and the man-made equipment that contains elements that modify the natural background by using the electrical mains. ${ }^{9}$

c. Close interpersonal interactions (to be discussed below). 
Geomagnetic influences are not in the conceptual realm of owners, builders and purveyors of working and living space in these modern times. The vast majority of people do not know about the role of the Birkland currents in creating:

A. The 3D grid matrix of transverse electromagnetic waves (TEM)

B. The $4.55 \mathrm{~Hz}$ resonance that living multicellular life forms are tied to in the structured water of their bodies

C. The placement within the 3D grid that has a direct affect upon the HBF. See the information at these links for a more in depth presentation relating to the $\mathrm{HBF} .{ }^{10,11}$

The environment is pervasively violated by the presence of the electrical power mains at alternating frequencies of $50 \mathrm{HZ}$ and $60 \mathrm{HZ}$ depending upon where you live. ${ }^{12}$ People work in conjunction with electrically powered equipment, much with the usage of electric motors. Most electric motors are not shielded and spray vast amounts of debilitating electrical and magnetic field components. The magnetic field components are especially debilitating.

Another workplace interface with your patient is the possible presence of hazardous materials (HazMat) used in a manufacturing processes. HazMat these days is usually under strict engineering controls and does not pose a direct threat to the biochemistry of a patient. HazMat of chemical origin is known to be the cause of peripheral neuropathy ${ }^{13}$ and liver cirrosis.

\section{Interpersonal interactions}

The person and the people that the patient spends most of their time with will give you a clue as to the possible nature of some aspects of HBF aberrations. A comparison of natal charts between partners will definitely show where the type and quality of stressor is being generated. The stressor may be physical as detailed by the respective aspects of both parties Mars \& Venus placement. Adverse aspects to the patients Jupiter cause emotional stress. Adverse aspects to the patients Mercury cause mental stress. Thus, the genetic allels as coded by the representations of the planetary aspects that determine the energetic underpinnings of the physical body show where the stressors become a problem. Work needs to be done to show the link between astrology and the meridian system.

\section{Life goals}

The reason that the patient comes to you is immediately for the relief of their symptomology. They may also come to you for assistance in losing weight, stopping smoking, guidance for diet and other mental/emotional issues. Most would like to be functioning at optimum capacity rather than some chronically reduced capacity. This is what you may do for them if they will only cooperate with your treatment protocol.

\section{Patient signs \& symptoms (S\&S)}

After a sufficient time for the above informational intake, it is relevant to request information as to just what is bothering the patient. This is followed by a back and forth discussion as to the nature of all of the S\&S that the patient may display of a mental, emotional and physical nature. The physical S\&S are comparatively easy to discern. The emotional and mental ones are not so straight forward. This is where your knowledge of Homeopathy will come in handy. You must become well acquainted with the protocol for finding the remedy needed for the symptoms displayed by your patient. The protocol will show you the kinds of questions that you should be asking and the relationship between mental/emotional disturbances to the HBF. In this manner, you may begin to make empirical correlations between patient symptoms and HBF tie-ins with the meridians/channels.

\section{Patient treatment}

Clinically you must seek to correct disharmony within the human body-field and/or remove discordant water structure harmonics. ${ }^{11}$ Both Homeopathy. ${ }^{14}$ and TCM are used in a patient treatment. Homeopathy was the first systemized western modality that utilized IM. Acupuncture ${ }^{15}$ was the first eastern and world modality that utilizes IM. One person shows that acupuncture is astrology with needles..$^{16}$ The usage of both modalities ensures a rapid return to health for your patient.

It is very important that you learn the difference between myth, ancient cosmological viewpoints, very ancient medical modalities and what modern science and empirical evidence coupled with practical clinical experience gives to you the clinician in these days and times. It is true that modern science has lifted the veil of so much ancient nonsense but still seems unable to grasp the basic tenets of IM. It does take some research to be able to separate the garbage from the hidden jewels within cultural expressions of healing modalities. ${ }^{17}$

Electro-acupuncture according to Dr. Voll (EAV) utilizes the meridian system of acupuncture and is very effective in the alleviation of patient symptoms. EAV is the basis of bioresonance therapy ${ }^{18}$ and the direct modern precursor to IM. EAV is the mode that all of the energy therapy systems utilize and is exemplified by the NutriEnergetics System. ${ }^{19}$ There are at least 8 different systems on the market today that utilize the pioneering work of Dr. Voll. ${ }^{20}$

Getting your patient to address their lifestyle choices is going to be your most challenging issue. Also, the anchoring that you do with your treatment will most likely not hold for long and the patient will need to come back again for another treatment in 3days, then 5 days and then weekly. After a couple to 3 weeks, they will need to come back monthly until you no longer see any further improvement physically and their emotional/mental S\&S abate and stabilize.

Anchoring is a difficult subject only in that the clinical efforts will be constantly subverted by the many sources of ubiquitous bioelectromagnetic pollution that exist in our modern times. Electrical motors are especially harmful and most factory processes are such as to encode inimical IM in to the foodstuffs being manufactured. Also, the desire of the manufacturers for your continued consumption of the product will be encoded into the foodstuff and unconsciously effect the continued buying and consumption of that product. Personal experience by the author has led him to suggest that at least $85 \%$ of all the products in grocery stores are harmful to your long term wellbeing. The specter of bio-electromagnetic pollution is very real both with manufactured foodstuffs and the vast array of consumer electronics.

The average treatment protocol only needs 15 to 20 seconds to make a positive effect. It is the anchoring that needs about 28 to 30 minutes to be effective due to patient expectations only. Patient expectations are a very powerful treatment in itself. The American Medical Association (AMA) has determined that $80 \%$ of patients that see MDs get better no matter what therapeutic modality is used with a patient. This is tied to the placebo effect. ${ }^{21}$. Unfortunately, many 
supposed medical experts see IM as a placebo effect simply because they do not understand the biophysics of IM. Also, ideational intent is a very powerful component of any mode of healing, make no mistake about that fact!

The use of the Cold Laser $^{22}$ i.e., low level laser, ${ }^{23,24}$ as a IM treatment modality is a lesser known one but gaining ground rapidly. Laser acupuncture relies upon similar dosage principles as needling. ${ }^{25}$, and knowledge of acupuncture theory and practice is required. ${ }^{26}$ Applied dosage is determined by manner of the intended effect, which is generally characterized as to stimulate or to sedate acupuncture points, Ashi Points, and Trigger Points. According to the Arndt-Schultz Rule, it can be seen that low dosages will stimulate and that higher dosages will sedate. Dosages of 0.5 to $2.5 \mathrm{~J} / \mathrm{cm}$ sq are reported to be effective in the stimulation of superficial acupuncture points, with 2.5 to $5.0 \mathrm{~J} / \mathrm{cm}$ sq being effective for deeper points and myofascial trigger points. ${ }^{27}$ Higher dosages $(8-12 \mathrm{~J} / \mathrm{cm} \mathrm{sq})$ are effective for the sedation of points.

The use of the cold laser is particularly suited for use with the 5 Shu points. ${ }^{28}$. The intent of the traditional acupuncture protocol is to stimulate the Mother point of the namesake point of the meridian for a deficiency in that meridian or conversely to reduce the Son for an excess. The Spleen Meridian will be used as an example, for instance as if there was an excess condition in the Spleen Meridian. Since the namesake Shu point on the Spleen meridian is the Earth/Shu-Stream point Sp3, the Metal/Jing-River point Sp5 is to be reduced. Sp5 is the Son of the namesake point Sp3. If there is a deficiency, the Mother of the $\mathrm{Sp} 3$ point is to be stimulated, $\mathrm{Sp} 2$. Using the laser to reduce means to apply a dosage of $8-12 \mathrm{Joules} / \mathrm{cm}$ squared. Conversely, a stimulatory dosage would be 0.5 to $5.0 \mathrm{Joules} / \mathrm{cm}$ squared. Thus to stimulate the Mother point of the namesake in the meridian treated, apply a dosage of 4 Joules/cm squared. In order to reduce the Son point of the namesake point in the meridian treated, apply a dosage of 10 Joules/cm squared. This protocol is in accordance with the ArndtSchultz Rule..$^{29,30}$

\section{A clinical protocol for monitoring \& harmonizing the HBF}

I have not yet found any suitable information concerning this topic online and thus the author described the protocol that he uses when treating a patient at this webpage. ${ }^{31}$

This protocol uses the HBF of the clinician to detect and harmonize the HBF disruptions within the HBF of the patient. This form of medical dowsing is not difficult to learn. The biggest issue is in maintaining a smooth, centered and balanced HBF while the clinician conducts the protocol upon the patient.

\section{Conclusion}

As you may understand by now, it is a very educated person that practices IM. However, you as the clinician must also be very much aware of the state of your own body-field. This includes the means of dealing with adverse energy fields that you will encounter from your patients. The author practices grounding in addition to washing the hands before and after every patient. In addition, you as the person of clinical practice ${ }^{32}$ will need to stay abreast of medical information, astrology, homeopathy, oriental medicine, acupuncture and life in general..$^{33}$ Those persons coming to you for assistance will need to be "patient" as you grow in depth and understanding of your chosen profession.

\section{Acknowledgments}

None.

\section{Conflict of interest}

The authors declare that there is no conflict of interest.

\section{Funding}

None.

\section{References}

1. Kolari PJ, Hietanen M, Nandelstad P, et al. Lasers in physical therapymeasurement of optical output power. Scand J Acupuncture and Electrotherapy. 1988.

2. Block EF. The Human Body Field. Journal of Informational Medicine. 2009.

3. Muxeneder R. Soft laser in the conservative treatment of chronic skin lesions in the horse. Der Prakt Tierarzt. 1987;68(1):12-21.

4. Ben Kavoussi. Acupuncture is astrology with needles. Science-Based Medicine. 2009.

5. Dyson M, Young S. Effect of laser therapy on wound contraction and cellularity in mice. Lasers in Medical Science. 1986;1(2):125-130.

6. Mester \& Mester. Wound Healing. Laser Therapy. 1989;1:7-15.

7. Airaksinen O, Rantanen P, Kolari PJ, et al. Effects of IR (904nm) and $\mathrm{He}-\mathrm{Ne}(632.8 \mathrm{~nm})$ laser irradiation on pressure algometry. Scand J of Acupuncture and Electrotherapy, TPs Paper to Nordic AP Society Annual Congress, Iceland, Europe. 1988;3:56-61.

8. Bruse E, Stuck. Measuring and reporting physical parameters in laser biomodulation research. In: Michal Schwartz, Michael Belkin, Editors, In Low Energy Laser Effects on Biological Systems. Proc SPIE. 1883, p. 21-26.

9. Valery EI, Alexander VP. Current Status of Low-Energy Laser Therapy in Russia:Search for Mechanisms, Clinical Applications and Equipment Development. In: Michal Schwartz \& Michael Belkin, Editors, In Low Energy Laser Effects on Biological Systems. Proc, SPIE 1883, p. 8-13.

10. Block EF. Structured Water. The Journal of Informational Medicine. 2011.

11. Block EF. Some Biological Implications Of the Giertz Shift. Journal Of Informational Medicine. 2012.

12. Trelles MA, Mayayo E, Mester A, et al. Low power lasertherapy:Experimental and clinical data. Scandinavian Journal of Acupuncture \& Electrotherapy. 1987;2:80-100.

13. Block EF. Peripheral Neuropathy as the Common Ground between Eastern and Western Medicine. Oriental Medicine Section. 2007.

14. Block EF. Homeopathy:The First Systematized Healing Modality Of Informational Medicine. Journal of Informational Medicine. 2007.

15. Block EF. Acupuncture as Informational Medicine. Journal of Informational Medicine. 2010.

16. Ben Kavoussi. Acupuncture is astrology with needles. Science-Based Medicine. 2009.

17. Karu T (1998) The Science of Low-Power Laser Therapy. Gordon \& Breach Science Publishers.

18. Biophysics. Journal Of Bioelectromagnetic Medicine. 2000.

19. http://www.neshealth.com/ 
20. http://www.biontology.com/wp-content/uploads/2012/08/Voll.pdf

21. http://en.wikipedia.org/wiki/Placebo

22. Rogers PAM, Janssens LAA, Jagger D. The efficacy of cold laser: A survey of members of the International Veterinary Acupuncture Society. 1987.

23. Philip AM Rogers. Clinical Uses of Low Level Laser Therapy. Medical Acupuncture. 1986.

24. Tunér J, Hode L. "Low Level Laser Therapy - Clinical Practice and Scientific Background”. USA: Prima Books; 1999.

25. Pontinen PJ (1995) Low Level Laser Therapy (LLLT) and laser acupuncture:a manual for physicians, dentists, physiotherapists and veterinary surgeons.

26. David Baxter G, Costas Diamantopoulos. Therapeutic Lasers: Theory and Practice. USA: Churchill Livingstone Edinburgh Medical; 1994. pp. 259.

27. Laakso. Plasma ACTH and Endorphin Levels in Response to LowLevel-Laser Therapy for Myofascial Trigger Points. Laser Therapy. 1994;6:133-142.
28. Block EF. Low-Level Laser Acupuncture Treatment In The Clinical Application Of The Five Shu Points. The Journal Ofbioelectromagnetic Medicine. 2010

29. Pontinen PJ. Mid-laser and TNS in back pain. Nordic AP Society Annual Meeting: Seminar on back pain; Oslo, Norway. 1987.

30. Grosman Z. Effect of laser irradiation on different cell structures. Sb Ved Pr Lek Fak Karlovy Univerzity. Hradci Kralove. 1976;19(34):375-388.

31. Components of the Electromagnetic Wave. Journal of Informational Medicine.

32. GD Baxter, AJ Bell, JM Allen, et al. Low Level Laser Therapy:Current Clinical Practice in Northern Ireland. Physiotherapy. 1991;77(3):171178.

33. Mester E, Mester AF, Mester A. The biomedical effect of laser application. Lasers in Surgery and Medicine. 1985;5(1):31-39. 